\title{
Circuit
}

Musiques contemporaines

\section{Avant-propos. Une hétérogenèse}

\section{Maxime McKinley}

Volume 29, numéro 2, 2019

URI : https://id.erudit.org/iderudit/1062562ar

DOI : https://doi.org/10.7202/1062562ar

Aller au sommaire du numéro

\section{Éditeur(s)}

Circuit, musiques contemporaines

\section{ISSN}

1183-1693 (imprimé)

1488-9692 (numérique)

Découvrir la revue

Citer ce document

McKinley, M. (2019). Avant-propos. Une hétérogenèse. Circuit, 29(2), 5-6. https://doi.org/10.7202/1062562ar

Ce document est protégé par la loi sur le droit d'auteur. L'utilisation des services d'Érudit (y compris la reproduction) est assujettie à sa politique d'utilisation que vous pouvez consulter en ligne.

https://apropos.erudit.org/fr/usagers/politique-dutilisation/
Cet article est diffusé et préservé par Érudit.

Érudit est un consortium interuniversitaire sans but lucratif composé de l'Université de Montréal, l'Université Laval et l'Université du Québec à Montréal. Il a pour mission la promotion et la valorisation de la recherche. https://www.erudit.org/fr/ 


\section{Avant-propos. Une hétérogenèse}

Maxime McKinley

Félix Guattari, discutant de la musique de Georges Aperghis, a avancé l'idée d'une «hétérogenèse dans la création musicale ${ }^{1}$ ». Il est très stimulant de penser la créativité en termes de différences interactives. Les «genèses » des numéros de Circuit procèdent presque toutes ainsi, et je profite de ce bref avant-propos pour faire le récit de quelques éléments hétérogènes ayant mis en branle la création de cette livraison. Cet exercice est une manière d'hommage à l'esprit de collaboration et au travail collectif qui anime la revue Circuit et, de facto, l'expression d'une gratitude envers ses artisans, parmi lesquels je compte les lecteurs.

Au moins six éléments hétérogènes ont sous-tendu la genèse de ce numéro:

1) Le premier catalyseur est venu en 2016, alors qu'Émilie Girard-Charest (contributrice de ce dossier) me demandait, peu après ma nomination à titre de rédacteur en chef de Circuit, ce qu'elle pouvait lire dans la collection au sujet de la microtonalité. Un peu embarrassé de répondre, force m'était de constater que sa question signalait une lacune, et je ne manquai pas d'en parler dès ma première réunion avec le comité de rédaction (le 7 avril 2016).

2) Au cours de cette même réunion, nous avions, entre autres choses, décidé d'inviter parmi nous un nouveau membre: Robert Hasegawa, spécialiste de microtonalité, professeur à l'Université McGill et codirecteur du présent numéro.

3) Réfléchissant à des dossiers que Hasegawa pourrait être invité à diriger pour Circuit, nous avions pensé à une monographie consacrée à Georg Friedrich Haas. Cette idée apparaît dans les notes de notre première réunion avec lui (le 14 juillet 2016) et s'est finalement mutée, ici, en un remarquable article de Landon Morrison.

4) Toujours au cours de cette période, Paul Bazin - doctorant en musicologie sous la direction du même Hasegawa, et qui n'était pas encore notre
1. Félix Guattari (2013),

"L’hétérogenèse dans la création musicale", Chimères, n ${ }^{0} 79$, p. 33-36. 
2. Ivan Wyschnegradsky (2013), Libération du son : écrits 1916-1979, textes réunis, présentés et annotés par Pascale Criton, Lyon, Symétrie. Voir le compte rendu de Sharon Kanach, paru en 2014 dans Circuit, vol. 24, $n^{\circ} 3$, p. 88-91.

3. En effet, le vol. $26, n^{\circ} 2$ était codirigé par Michel Duchesneau et Annelies Fryberger, tandis que le vol. 26, $n^{0} 3$ l'était par Serge Cardinal et Solenn Hellégouarch.

4. Rappelons que lanza écrit son nom sans majuscules.

5. Ici, le concert de la SMCQ ayant eu lieu le 23 janvier 1975 permet de rattacher quelques fils: Iors de ce concert, Bruce Mather et son épouse, Pierrette Lepage, interprétaient, notamment, les Intégrations, op. 49 (1967) de Wyschnegradsky et, en création, plectros IV (1974) de lanza. À ce sujet, voir : http://smcq. qc.ca/smcq/fr/evenement/21563/ Concert_65_Robert_Leroux (consulté le 7 avril 2019). Quelques mois avant ce concert, dans une lettre datée du 6 août 1974, Wyschnegradsky "priait" Mather de bien vouloir "saluer Mr. alcides lanza", avant de décrire ses liens avec l'Argentine et de les comparer avec ceux de la France (évoquant au passage Juan Carlos Paz, Pierre Schaeffer et Claude Ballif). Lettre de Wyschnegradsky à Mather, 6 août 1974. Fonds Bruce Mather (Fo402), Correspondance avec Ivan Wyschnegradsky, boîte 15 , filière 26, Archives de l'Université de Calgary. Je remercie Paul Bazin pour ces informations.

6. Au sujet de Takasugi, voir: www. steventakasugi.com/ (consulté le 16 avril 2019).

7. Voir: www.nohaybanda.ca/nhb11. html (consulté le 7 avril 2019). coordonnateur administratif et secrétaire de rédaction - portait à mon attention le fait que Bruce Mather, disciple d'Ivan Wyschnegradsky et pionnier de la microtonalité «ultrachromatique» (non seulement au Québec, mais, plus largement, en Amérique du Nord et au-delà), célébrerait ses 80 ans en 2019. Sensible à l'importance de ne pas oublier les apports des vétérans, j’en avais pris bonne note.

5) Comble de synchronisme, le comité de rédaction et moi mettions alors en place un corpus d'évaluateurs externes pour la revue, incluant la compositrice Pascale Criton, aussi spécialiste de microtonalité et éditrice des écrits de Wyschnegradsky². Elle est interviewée par Sharon Kanach dans les pages qui suivent.

6) Par ailleurs, dans le volume 26, nous avions coup sur coup publié des dossiers codirigés par des duos de professeurs universitaires et de doctorants ${ }^{3}$. Il y avait là une belle occasion d'aller de nouveau en ce sens, avec le tandem formé par Hasegawa et Bazin.

Finalement, ces fils hétérogènes (et sans doute beaucoup d'autres) sont à l'origine de ce que vous vous apprêtez à lire. Cette «hétérogenèse » n'a pas pour autant donné lieu à un résultat hétéroclite ou décousu. Au contraire, la thématique prise en charge par les directeurs invités traverse la quasi-totalité du numéro, lui donnant beaucoup d'unité. Peut-être les deux exclusivités web de la rubrique Actualités sont-elles un peu plus exogènes, mais là encore, il ne faut pas sous-estimer la richesse des concordances latentes. Ainsi, le pianiste Daniel Áñez a signalé que le compositeur alcides lanza ${ }^{4}$ célébrerait ses 90 ans en 2019, ce qui n'est pas sans écho avec le $80^{\mathrm{e}}$ anniversaire de Mather ${ }^{5}$. Nous avons convenu que Daniel s'occuperait lui-même de le souligner, et que cela prendrait une forme essentiellement audiovisuelle, en ligne. Quant à l'autre exclusivité web, l'élément déclencheur fut un appel téléphonique de Charles-Antoine Fréchette (déjà contributeur de ce numéro), afin de nous signaler la venue à Montréal de Steven Kazuo Takasugi ${ }^{6}$, dans le cadre de la présentation de Sideshow (2009-2015) dans la série de concerts No Hay Banda - un événement auquel participaient, entre autres, Áñez au piano et GirardCharest au violoncelle?

En somme, c'est une belle chose qu'une revue puisse être l'interface rendant possible une telle «topologie chimique ». Mais cette « hétérogenèse » n'a pas fini de prendre de l'expansion, et c'est ici que vous intervenez. En effet, plus il y a de lecteurs différents, mieux c'est! Cela «au profit d'une hétérogenèse qui dissout l'individuation d'énonciation. On ne sait plus au juste ce qui circonscrit l'énonciation : l'ensemble orchestral est un agencement collectif auquel appartient également le public ${ }^{8}$.» 